\title{
Occurrence Analysis of Cutaneous Leishmaniasis particularly in Pakistan
}

\author{
Hajra Haneef ${ }^{2 *}$, Nazar-ul-Islam ${ }^{1}$, Khalid Mehmood ${ }^{1}$ and Sajid Ali ${ }^{1}$ \\ ${ }^{1}$ B.P.D, N.I.H. Chak shahzad, Pakistan \\ ${ }^{2}$ Ex-Lecturer of College of Medical Laboratory Technology, Pakistan
}

*Corresponding author: Hajra Haneef, Ex-Lecturer of College of Medical Laboratory Technology, Pakistan

\section{ARTICLE INFO}

Received: 幽 July 13, 2019

Published: July 19, 2019

Citation: Hajra Haneef, Nazar-ul-Islam, Khalid Mehmood, Sajid Ali. Occurrence Analysis of Cutaneous Leishmaniasis particularly in Pakistan. Biomed J Sci \& Tech Res 19(5)-2019. BJSTR. MS.ID.003359.

\section{Review}

Cutaneous leishmaniasis (CL) which is endemic in the tropics and neotropics Muhammad et al. [1], in Iran Iraj et al. [2], in the Middle East and North Africa including Yemen Mohammed et al. [3]. It is a major public health problem in the country especially alongside regions bordering the neighboring Afghanistan and cities that have had the maximum influx of refugees Hepburn NC [4]. In Pakistan, CL is a public health burden and is considered a Neglected Tropical Disease. It can cause severe physical disfigurement that leads to stigmatization and discrimination. Yet, no official data is available about the number of people infected by the disease in the country Medecins SF [5]. The burden of CL in terms of morbidity and social stigmas are concentrated on certain developing countries in Asia, Africa, and South America Fariborz et al. [6]. It is often referred to as a group of diseases because of the varied spectrum of clinical manifestations, which range from small cutaneous nodules to gross mucosal tissue destruction Richard et al. [7]. (CL) is an immunemediated skin pathology caused mainly by Leishmania (L.) major, Leishmania tropica, Leishmania braziliensis, L. mexicana, and L. amazonensis Fariborz et al. [6].

Initial symptoms include skin lesions, which develop after several weeks or months after being infected, and swollen glands. The lesions - closed or open sores - can change overtime in size and appearance. They are usually painless but can become painful if infected with bacteria. The lesions can take a long time to heal and usually leave scarring Pubmed [8]. CL is responsible for chronic and disfiguring skin lesions resulting in morbidity and social stigma Celine et al. [9] Among parasitic diseases, morbidity and mortality caused by leishmaniasis are surpassed only by malaria and lymphatic filariasis Caryn et al. (2008). Worldwide, leishmaniasis causes 70,000 deaths per year Edoardo et al. [10]. In 2016, 87 of the 200 (44\%) countries that reported their status to the World Health Organization (WHO) were considered endemic for cutaneous leishmaniasis (CL), and 7 (4\%) were endemic for visceral leishmaniasis (VL). The Eastern Mediterranean Region (EMR) had the highest proportion of CL-endemic countries (82\%), followed by the Region of the Americas (AMR) with 58\%. For VL, the "leader" was again EMR (82\% of countries), with 51\% of European Region (EUR) and $45 \%$ of South-East Asia Region (SEAR) countries meeting this designation Deresinski S [11].

Children are most commonly affected victims in Satluj Valley of Himachal Pradesh. Over a period of one year 337 cases of CL were registered, out of them 115 children (0-18 years) were enrolled in this study, in 2018. School going (6-18 years) population was predominantly affected age group. Males outnumbered the 
females with a ratio of 1.88. Face was most commonly affected site and most (65.21\%) cases presented with single lesion. Nodules (40.87\%), plaques (24.35\%) and papules (7.39\%) were commonly observed morphologies although mixed patterns and atypical forms were also found. Most (62.60\%) cases presented within 1-3 months of lesion onset. All cases responded well to treatment with antimonials. Lesional pain, swelling, bleeding, pigmentation and scarring were minor post treatment side effects. Pancham $\mathrm{K}$ et al. [12]. Leishmaniasis in Yemen is still not fully investigated nor well studied. Outbreaks of CL in western highland declared. However, there are no reports concerning the disease and the circulating species in the region.

The aim of this study was to determine the prevalence of CL in Utmah district located in Western Highlands in Yemen. A crosssectional survey was carried out at those highlands. For the survey, 1165 participants were subjected to Leishmanin Skin Test (LST) accompanied with direct interviews and physical examination. The overall prevalence of CL in the district was $18.5 \%$ and the CL was more frequent in the escarpments with a prevalence of $37 \%$, including $5.5 \%$ for active lesion and $31.5 \%$ for scar of healed lesions. Children under the age of 16 years old comprised most of the CL cases (76.3\%). According to this investigation the escarpments of western highlands in Yemen were hyper endemic areas for CL and the infection was more prevalent in children. Mohammed MA et al. [3]. CL is endemic in Pakistan and is widely spreading day by day. Leishmaniasis is endemic in Jacobabad, Dadu and Larkana districts of Sindh Province. It was pointed out that the disease is dramatically spreading in the country and warned that if the preventive measures were not taken at right time there would be a serious public health problem in the country.

One study was conducted in the Department of Dermatology, Chandka Medical College Larkana. A total of 1640 cases of CL were seen during 2004 to 2005 . Among the 1640 patients, 470 were residents of different cities of Balochistan Province; and 1170 were the residents of different cities of Sindh Province like Jacobabad, Ratodero, Shahdadkot, Qambar, Warah, Mehar, Dadu, Dokri and Larkana. All the patients were aged between 3 months and 60 years. Seven hundred eighty were males and 860 were females. Duration of the disease ranged from 1 to 12 months. Most of the patients had single lesions but multiple lesions were also seen on the exposed (mainly) and unexposed parts of the body. Clinically, the lesions were classified as dry ulcerative, wet ulcerative, dry papular, nodular, and crusted lesions. Diagnosis was made on the basis of smear test and clinical presentation of the skin lesions. All the cases were treated with the meglumine antimoniate $600 \mathrm{mg} /$ day (adults) and $15 \mathrm{mg} / \mathrm{kg} /$ day (children) either intramuscularly for 20 consecutive days or intralesionally for 10 alternate days. The molecular characterization and other studies are needed for further evaluation of this disease. Abdul MB et al. [13].

CL is known for its clinical diversity and increasing numbers of new and rare variants of the disease are being reported these days. The aim of this descriptive study was to look for and report the atypical presentations of this common disease occurring in Pakistan. The study was carried out in three hospitals $\mathrm{MH}$, Rawalpindi; PAF Hospital, Sargodha; and CMH, Muzaffarabad) from 2002 to 2006. Military and civilian patients of all ages, both males and females, belonging to central and north Punjab province and Kashmir were included in the study. Clinical as well as parasitological features of cutaneous leishmaniasis were studied. The unusual lesions were photographed and categorized accordingly using simple descriptive statistics. Out of 718 patients of cutaneous leishmaniasis, 41 (5.7\%) had unusual presentations. The commonest among unusual morphologies was lupoid leishmaniasis 14 (34.1\%), followed by sporotrichoid 5 (12.1\%), paronychial 3 (7.3\%), lid leishmaniasis 2 (4.9\%), psoriasiform 2 (4.9\%), mycetoma-like 2 (4.9\%), erysipeloid 2 (4.9\%), chancriform 2 (4.9\%), whitlow 1 (2.4\%), scar leishmaniasis 1 (2.4\%), DLE-like 1 (2.4\%), 'squamous cell carcinoma'-like 1 (2.4\%), zosteriform 1 (2.4\%), eczematous 1 (2.4\%), verrucous 1 (2.4\%), palmar/plantar $1(2.4 \%)$ and mucocutaneous $1(2.4 \%)$.

In Pakistan, an endemic country for CL, the possibility of CL should be kept in mind while diagnosing common dermatological diseases like erysipelas, chronic eczema, herpes zoster, paronychia; and uncommon disorders like lupus vulgaris, squamous cell carcinoma, sporotrichosis, mycetoma and other deep mycoses. Arfan B [14].

CL is the disease of a public health concern in Punjab, Pakistan. The knowledge of how the population perceives the disease and its vector is essential in order to design an effective management strategy, but such studies are rare in Pakistan. The present study was based on a cross-sectional self-administered survey comprising 250 household samples collected from five localities including Bhawalpur, Multan, Jhang, Faisalabad and Lahore. The results revealed that the respondents had a poor knowledge of the vector and disease. Few of the respondents were aware about the identification of sand flies, their breeding place, biting time, transmission of leishmaniasis and control measures. Skin infection and sandflies as the main disease symptom and vector of the disease, respectively, were known to some of the respondents.

Some believed that summer was the main peak incidence of the disease and it could be transmitted from man to man via contact. However, most of the respondents believed that the disease could be cured. Admission to hospitals, cleanliness and use of bed nets were the treatment measures for the disease in suspected patients, whereas some thought that the use of bed nets could be helpful in preventing leishmaniasis infection.Poor knowledge of the disease and its vector in the study population emphasize the need to initiate health education and awareness campaigns to minimize the risks of CL outbreaks in the future. Ayesha A et al. [15]. This study primarily aimed to identify the causative species of cutaneous leishmaniasis (CL) in the Khyber Pakhtunkhwa Province of Pakistan and to distinguish any species-specific variation in 
clinical manifestation of CL. Diagnostic performance of different techniques for identifying CL was assessed. Isolates of Leishmania spp. were detected by in vitro culture, polymerase chain reaction (PCR) on DNA extracted from dried filter papers and microscopic examination of direct lesion smears from patients visiting three major primary care hospitals in Peshawar.

A total of 125 CL patients were evaluated. Many acquired the disease from Peshawar and the neighboring tribal area of Khyber Agency. Military personnel acquired CL while deployed in north and south Waziristan. Leishmania tropica was identified as the predominant infecting organism in this study (89.2\%) followed by Leishmania major (6.8\%) and, unexpectedly, Leishmania infantum (4.1\%). These were the first reported cases of CL caused by $\mathrm{L}$. infantum in Pakistan. PCR diagnosis targeting kinetoplast DNA was the most sensitive diagnostic method, identifying $86.5 \%$ of all samples found positive by any other method. Other methods were as follows: ribosomal DNA PCR (78.4\%), internal transcribed spacer 2 region PCR (70.3\%), culture (67.1\%), and microscopy (60.5\%). Clinical examination reported 14 atypical forms of CL. Atypical lesions were not significantly associated with the infecting Leishmania species, nor with "dry" or "wet" appearance of lesions. Findings from this study provide a platform for species typing of CL patients in Pakistan, utilizing a combination of in vitro culture and molecular diagnostics. Moreover, the clinical diversity described herein can benefit clinicians in devising differential diagnosis of the disease. Nazma HK et al. [16].

One study in 2017 in Dir Lower District showed the overall incidence of CL was $21.1 \%$. The prevalence was observed to be significantly higher among Afghans than the local population (32.5\% vs. $20.3 \%$, OR: $1.6, \mathrm{p}<0.05$ ). The distribution of CL showed significant difference with respect to all studied demographic parameters, except subject's age and tehsil. More than half of the infected patients $(56.8 \%)$ had wet lesions and majority of the infected patients (42.3\%) had only one lesion. Moreover, face and arms were the most frequently inflicted body parts. It was further observed that among various antimonials used by the patients, sodium stibogluconate demonstrated the highest efficacy followed by glucantim and stibotim whereas thermotherapy showed no efficacy. Hadayat R [17]. In Pakistan, CL is widely distributed in endemic areas. One study conducted in Multan during 2018, reported outbreak of CL in 14 out of the 68 Union Councils of Multan.

The material for diagnosis of CL was obtained from 461 patients from 239 clinical laboratories and health care centers in Multan. Of these patients, 42.9\% (198 of 461) having positive findings of amastigotes in Giemsa stained tissue impression smears. The distribution of CL skin lesions was highly variable. Most patients (76.2\%) had a single lesion. Most CL lesions were found on a single hand $(23.7 \%)$. The prevalence of two CL lesions per patient was $21.7 \%$, while three or more positive CL lesions were observed in $2.0 \%$ of patients. The $198 \mathrm{CL}$-positive patients ranged in age 10-40 yr old, 101 were males, and 97 were females. Logistic regression analysis of leishmaniasis versus age and gender showed that both the age and gender had a significant $(\mathrm{P}<0.05)$ effect on the occurrence of infection. Muhammad MA et al. [1]. One study in 2016 , showed that eyelid is rarely involved possibly because the movement of the lids impedes the sand fly from biting the skin in this region. This study reports 6 rare cases of eyelid CL. The patients were diagnosed by skin scraping, culture, and PCR from the lesions.

Skin scraping examination showed Leishmania spp. amastigotes in the cytoplasm of macrophages. Culture examination was positive for Leishmania spp. PCR was positive for Leishmania major and Leishmania tropica. The lesions were disguised as basal cell carcinoma, chalazion, hordeolum, and impetigo. The patients were treated with intramuscular meglumine antimoniate $(20 \mathrm{mg} / \mathrm{kg} /$ day) for at least 3 weeks. They showed a dramatic response, and the lesions almost completely disappeared. These findings emphasized the importance of clinical and diagnostic features of lesions, characterized the phylogenetic relationship of isolated parasites, and reviewed the literature on ocular leishmaniasis. Iraj MP et al. [2]. This study presented seven rare cases of lip leishmaniasis in Iran. Tissue impression smear, culture, PCR and phylogenetic analysis were carried out for explicit diagnosis. Skin scraping investigation showed several Leishmania spp. amastigotes in the cytoplasm of macrophages. Culture examination was positive for Leishmania spp. PCR was positive for L. major, L. tropica, and L. infantum. Differential diagnosis includes orofacial granulomatosis, basal cell carcinoma, squamous cell carcinoma, and mesenchymal tumors.

The cases were treated with systemic meglumine antimoniate (Glucantime). No relapses were observed during 1 year of followup. Early detection of the infection is necessary in order to start effective treatment and prevent more serious complications. Iraj MP et al. [18]. In 2014 epidemiological data from 2457 patients were retrieved from the local WHO suboffice. Shapefile layers of districts, cities, villages, land cover, soil type and digital elevation model (DEM) of the Herat province were used to assess, by logistic regression modelling, the effects of land cover, soil types, elevation, and proximity to the Harirud river on the distribution of ACL. The key determinants of distribution were: (i) close proximity to the Harirud river, (ii) elevation between 700 and 1200 m, (iii) intensive and intermittent irrigated cultivated land, and (iv) Haplocalcids with Torriorthents and Torrifluvents soil types. No ACL cases were found below700 m,and a few cases were presentat $>1200 \mathrm{~min}$ irrigated areas around the Harirud river Medecins SF [19].

These findings suggest that moist soil and the humidity from irrigated areas found between 700 and $1200 \mathrm{~m}$ provide suitable breeding sites of Phlebotomus sergenti, the main sandfly vector of L.tropica in Afghanistan. The effect of elevation also explains the predominance of ACL over ZCL in this region. The present study showed that distribution of ACL is strongly associated with environmental factors in West Afghanistan where the political and socio-economic conditions may also affect the epidemiology of CL. 
Mahdi F et al. [20]. One paper publish in 2019, according to it despite the known burden of neglected tropical diseases (NTDs) on child health, there is limited information on current efforts to increase pediatric therapeutic options. Objective of study was to quantify and characterize research activity and treatment availability for NTDs in children in order to inform the prioritization of future research efforts. In this study, a review of the World Health Organization's (WHO) International Clinical Trials Registry Platform to assess research activity for NTDs. The burden of disease of each NTD was measured in terms of disability adjusted life years (DALYs), which was extracted from the Global Health Data Exchange.

First- and second-line medications for each NTD were identified from WHO guidelines. We reviewed FDA drug labels for each medication to determine whether they were adequately labeled for use in children. Descriptive statistics, binomial tests, and Spearman's rank order correlations were calculated to assess research activity compared to burden of disease. Children comprised $34 \%$ of the 20 million DALYs resulting from NTDs, but pediatric trials contributed just $17 \%(63 / 369)$ of trials studying these conditions ( $\mathrm{p}<0.001$ for binomial test). Conditions that were particularly under-represented in pediatric populations compared to adults included rabies, leishmaniasis, scabies, and dengue. Pediatric drug trial activity was poorly correlated with pediatric burden of disease across NTDs (Spearman's rho $=0.41, p=0.12$ ). There were 47 medications recommended by the WHO for the treatment of NTDs, of which only $47 \%(n=22)$ were adequately labeled for use in children. Of the 25 medications lacking adequate pediatric labeling, three were under study in pediatric trials. Writers concluded that there is a substantial gap between the burden of disease for NTDs in children and research devoted to this population. Most medications lack adequate pediatric prescribing information, highlighting the urgency to increase pediatric research activity for NTDs with high burden of disease and limited treatment options Chris AR et al. [21].

\section{References}

1. Muhammad MA, Mufasser M, Ullah N, Zaman A, Akbar A, et al. (2018) Cutaneous Leishmaniasis in the Metropolitan City of Multan, Pakistan, a Neglected Tropical Disease. Journal of Medical Entomology 55(4): 10401042 .

2. Iraj M P, Hossein M, Handjani F, Reza G (2016) Cutaneous Leishmaniasis of the Eyelids: A Case Series with Molecular Identification and Literature Review. The Korean Journal of Parasitology 54(6): 787-792.

3. Mohammed M, Mohamed Suleiman, Awad G, Ahmed M (2019) Prevalence of Cutaneous Leishmaniasis in Western Highlands in Yemen. Journal of Tropical Medicine 8248916
4. Hepburn NC (2003) Cutaneous leishmaniasis: an overview. JPGM 49(1): 50-54.

5. Medecins SF (2018) TROPICAL \& NEGLECTED DISEASES, Cutaneous leishmaniasis in Pakistan.

6. Fariborz B, Harandi MA, Rafati S, (2018) Biomarkers of Cutaneous Leishmaniasis. Front Cell Infect Microbiol 8: 222

7. Richard R, Brooker S, Dujardin JC, Louzir H, Pirmez C, et al. (2007) Review Cutaneous leishmaniasis. The Lancet Infectious Diseases 7(9): 581-596.

8. Pubmed mail (2019) Pakistan General Health Risks: Leishmaniasis OUTBREAK Leishmaniasis is highly endemic in the tribal areas. Recent cases have been reported in Khyber Pakhtunkhwa

9. Celine A, Vink M, Jalal S, Nahzat S, Picado A, et al. (2019) Cost Effectiveness of New Diagnostic Tools for Cutaneous Leishmaniasis in Afghanistan. Appl Health Econ Health Policy 17(2): 213-230.

10. Edoardo T G, Romano M, Ruiz J, Arenas R (2017) Leishmaniasis: a review. F1000 Research 6: 750.

11. Deresinski S (2019) Leishmaniasis in the European Region and the World. Clinical Infectious Diseases 68: 3.

12. Pancham K, Garg A, Kumari S, Negi A (2018) One-year prospective study of pediatric cutaneous leishmaniasis: a neglected tropical disease in sub Himalayan region, India. Int J Contemp Pediatr 5(4): 1546-1550.

13. Abdul M, Rahman F, Katakura Ken (2008) Journal of Pakistan Association of Dermatologists, Leishmaniasis in Sindh, Pakistan: Outbreak and review of the literature. Journal of Pakistan Association of Dermatologists 18(4): 212-219.

14. Arfan B, Simeen BR (2008) Many faces of cutaneous leishmaniasis. IJDVL 74(1): 23-27.

15. Ayesha A, Aazhar H, Qadir A, Makhdoom A (2015) A Cross-Sectional Survey of Knowledge, Attitude and Practices Related to Cutaneous Leishmaniasis and Sand Flies in Punjab, Pakistan. Plos 10(6): e0130929.

16. Nazma HK, Bari A, Hashim R, Khan I, Muneer A (2016) Cutaneous Leishmaniasis in Khyber. Pakhtunkhwa Province of Pakistan: Clinical Diversity and Species-Level Diagnosis. Am J Trop Med Hyg 95(5): 11061114

17. Hadayat R, Atta R (2017) Cutaneous leishmaniasis in Dir Lower District, NorthWest Pakistan: epidemiology and treatment response. Journal of Pakistan Association of Dermatologists 27(4): 353-362.

18. Iraj MP, Hossein M, Handjani F, Reza G (2017) Lip leishmaniasis: a case series with molecular identification and literature review. BMC Infectious Diseases 17: 96.

19. Medecins SF (2018) TROPICAL \& NEGLECTED DISEASES Cutaneous leishmaniasis in Pakistan.

20. Mahdi F, Karamianb M, Amin M, Taylore RW Ghoheg HP, Abobakar S, et al (2014) Distribution pattern of anthroponotic cutaneous leishmaniasis caused by Leishmania tropica in Western Afghanistan during 20132014. Acta Trop 176: 22-28.

21. Chris AR, Hotez P, Monuteax, Niescierenko M, Bourgeosis T, et al. (2019) Neglected tropical diseases in children: An assessment of gaps in research prioritization. PLOS. 
ISSN: 2574-1241

DOI: 10.26717/BJSTR.2019.19.003359

Hajra Haneef. Biomed J Sci \& Tech Res

(CC) This work is licensed under Creative

Submission Link: https://biomedres.us/submit-manuscript.php

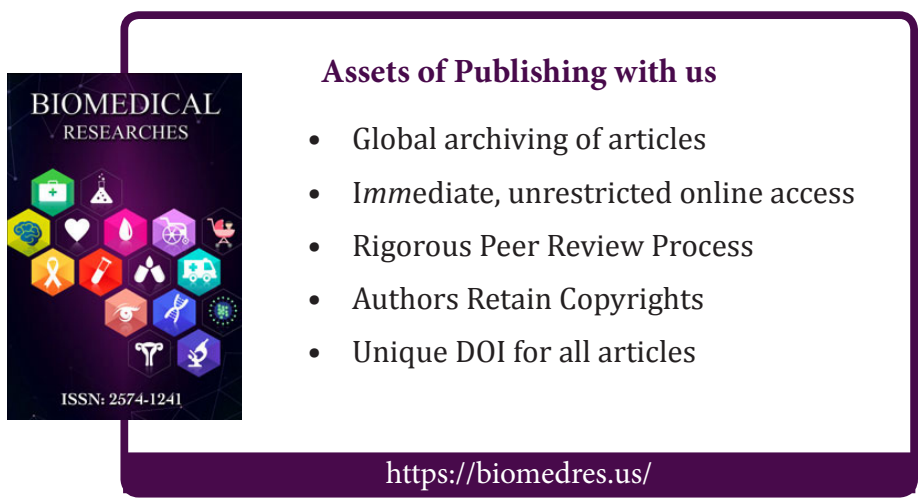

